\title{
THE IMPACT OF STREET PROPERTIES ON COGNITIVE MAPS
}

\author{
Mahsan Mohsenin ${ }^{a}$, Andres Sevtsuk ${ }^{b}$ \\ Massachusetts Institute of Technology, 77 Massachusetts Ave, Cambridge, MA 02139, USA \\ E-mails: ${ }^{a}$ mohsenin@alum.mit.edu (correspondingauthor); ${ }^{b}$ andres_sevtsuk@sutd.edu.sg
}

Received 03 October 2013; accepted 05 November 2013

\begin{abstract}
This paper investigates the relationship between street properties and cognitive maps. It is focused on the question of how human cognition of the built environment is affected by street properties. Building on the foundations of Kevin Lynch's studies of environmental perception (Lynch 1960) and recent configuration measurement techniques of the built environment, it addresses an important question that Lynch has left unresolved: Why do people have more complete recollections of some parts of the urban environment, and not others?

This paper proposes an analytical measurement framework based on graph theory to compare the results of cognitive maps with objective spatial properties of the corresponding built environment. In order to test our hypothesis, street geometry is measured and defined based on graph theory in two selected areas with similar geometries in Kenmore, Boston and Kendall Sq., Cambridge, MA. Cognitive maps are then collected based on specifically designed map drawing surveys. Finally, the relationship between graph results and cognitive maps is examined in order to identify the ways that street properties affect human perception.
\end{abstract}

Keywords: configuration patterns, street properties, cognitive maps, geometrical properties.

Reference to this paper should be made as follows: Mohsenin, M.; Sevtsuk, A. 2013. The impact of street properties on cognitive maps, Journal of Architecture and Urbanism 37(4): 301-309.

\section{Introduction}

The relationship between spatial structure of the built environment and social behavior has always been one of the central debates in urban planning. Among different aspects of the built environment, studies on cognitive maps and urban travel found that people's cognition is affected by urban configuration; however, the relationship between street properties and perception has not been much explored. Kevin Lynch studied people's perception based on certain characteristics of the city to explore how people remember paths, edges, districts, nodes and landmarks. Lynch's research focused on large neighborhoods or whole districts of a city. This paper takes a closer look at particular locations in Boston. Rather than focusing on a large set of two-dimensional attributes of urban form, it takes a more nuanced look at geometrical attributes of streets and their impact on cognitive maps. This research does not account for land use and design properties of streets, nor other people's social presence on the street.

\section{Cognitive mapping}

Cognitive mapping is known as a spatial problem-solving method, since it represents how people translate their environment into maps. Downs and Stea explain cognitive mapping as a psychological process, by which individuals recall, code and decode their built environment (Sanoff 1991). "The knowledge of cognitive mapping includes the knowledge of routes and the location of key environmental features" (Sanoff 1991: 76). In this research, objective properties of streets are tested to evaluate their impact on the knowledge of routes.

We argue along with Lynch that there could be an important intermediary step between actual street patterns and cognitive maps where the geometric characteristics of street networks affect cognitive maps. Allegedly, this paper claims that people perceive their built environment based on some "biases", while not all theories accept the impact of "biases". This research tries to find out one of these "biases" in the impact of street properties on people's perception. 
The methodology used in this study is grounded in the comparison between the completeness of cognitive maps with objective properties of streets in order to see if particular properties of streets are remembered more completely. To test the completeness of cognitive maps, compiled maps are produced as the result of cognitive map surveys, which asked people to draw in a boundary with streets based on their memory.

\section{Cognition of street structures}

In spite of several attempts to understand environmental cognition, the question of people's memory of a place has always seemed problematic because of the gap between urban planners and human cognition researchers. Space Syntax analysts made a significant contribution to advance configurational understanding of the built environment (Penn 2003; Conroy 2003; Whitehand 1987), whereas space syntax does not take into account the role of visual perception. Alan Penn in his exploration of spatial cognition, made an attempt to derive a non-metric space from metric visibility graphs (Penn 2003). In order to focus on cognition of street structures, this research tries to explore the correlation between street properties and cognitive mapping.

In space syntax, there is a focus on way-finding and people's movement (Hillier 1996; Penn 2003), while this study aims to signify the role of geometrical properties of streets. Appleyard used sequential photographs to capture the perception of streets for non-pedestrian (Appleyard et al. 1964), while none of these studies analyzed the impact of street properties on people's perception. In this research, therefore, the cognitive disposition of people is mapped based on street structures in order to evaluate how human cognition of the built environment is affected by street properties.

\section{Research design and hypotheses}

In the objective framework of this research, street geometry is defined and measured based on graph theory in two selected areas. Cognitive maps are then collected based on specifically designed map drawing surveys. Finally, the relationship between graph results and cognitive maps is examined to identify the ways that street properties affect human perception. In order to examine the impact of street properties on cognitive mapping, this research is inspired by looking at places where Kevin Lynch investigated in Boston and as a result, two areas with similar geometrical properties are selected in Kenmore and West Back Bay compared to Kendall Sq. These areas both have continuous streets, well-defined street patterns and angled streets (Fig. 1). In order to control the effect of other factors, selected survey areas are chosen with homogenous distribution of land use services.

This paper investigated different assumptions about the relationship between street properties and people's perception to test them within a comparison of the actual measurement of streets and the empirical results. The following points delineate the evolution of tested hypothesis in this research.

Hypothesis 1: People will remember continuous paths more clearly than interrupted paths. Looking at previous studies about street continuity by Kevin Lynch (Lynch 1960: 106) and the use of axial lines by space syntax (Hillier 1996: 132) brought up this hypothesis. Continuity is defined based on the sight-line for streets not being interrupted by buildings and not having an angular change of more than 20 degrees (Hillier 1996; Figueiredo, Amorim 2005). People's mind will lose its sense of orientation after a certain number of directional changes on the path. As a result, people can remember continuous paths more easily, since they can better orient themselves with limited number of orientation changes. The second reason
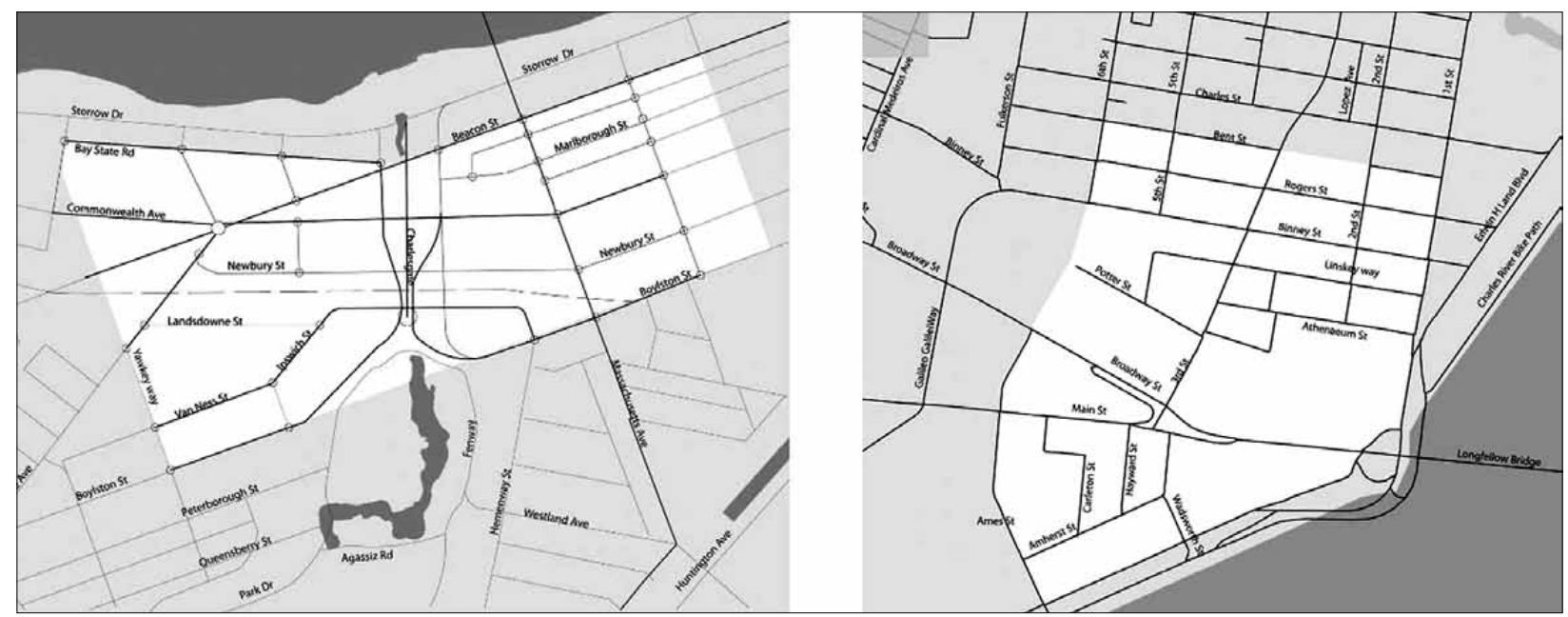

Fig. 1. Left: Kenmore and West Back Bay survey Area / Right: Kendall Sq. Survey Area 
to support this argument relies on the fact that continuous streets consist of several short paths. People thus use them a lot, and therefore remember them well.

Hypothesis 2: People will remember wider streets more easily than narrow streets. The first reason to support the idea of a clearer image of wider streets comes from the fact that the width of streets is designed in regard to its frequency of use and as a result, people can better remember wider streets. The second reason to support this idea relies on better street perspectives that people can encode in their minds from wider streets, as they have a broader image of their surroundings. It might be argued that continuity and street width are correlated. While we will test this correlation, we claim street width as a separate hypothesis influencing people's perception, because wider streets are not necessarily the most continuous ones and we can test their impact separately.

Hypothesis 3: People will remember streets with more number of intersections (density of intersection). The density of intersection is defined as the number of intersections along the total length of a street. Lynch's idea about nodes as decision points and way-finding ideas (Gell 1985; Golledge, Garling 2003) inspired me to test this hypothesis as a street property. First, I argue that the number of junctions influences the quality of access to other nodes, which can result in more frequency of use. Second, streets with more number of junctions will be better remembered, because every junction is a decision point, which makes people think about their options and as a result can be better remembered.

Hypothesis 4: Well-defined street patterns are remembered much easier than non-defined patterns. Based on studies of Gell (1985), Istomin and Dawyer (2009) on way-finding and the genesis of Marshall's studies on street taxonomies (Marshall 2005), We argue that people encode the geometric structure of the built environment in their mind in places with well-defined patterns. For instance, streets designed with a clear pattern such as a grid or a star-shape can be better perceived compared to non-defined patterns.
Hypothesis 5: People will better remember junctions with less angular deviation from perpendicular angles. Previous researchers have found that perpendicular angles are more easily remembered (Sadalla, Montello 1989). As a result, people can recall angles based on the nearest perpendicular angle. For instance, if angle between two streets is 80 degrees, people can recall it as it is near 90 degrees, while remembering a deviation of 45 degrees is more challenging. Studies on angularity and route selection (Sadalla, Montello 1989; Conroy 2003) provided the onset of this hypothesis.

\section{How to measure hypotheses and compare results with cognitive maps?}

The key question to this methodology is to understand how to measure each hypothesis to then compare it with empirical results. To measure this research hypothesis about street properties, it is significant that each hypothesis be measured exactly and unambiguously through mathematical representation of street properties to provide reliable evaluations based on the geometric analysis and survey results.

\section{Continuity}

The first hypothesis claims that people will remember continuous paths more clearly than interrupted streets. Bill Hillier has measured continuity based on axial lines representing the sight-line for each street (Hillier, Hanson 1984: 95-97; Hölscher et al. 2007). We argue that sight-line cannot measure continuity in this study, because the sight-vision has its shortcomings compared to intuitive perception of the people. As a result, in this research, the length of streets is measured where neither it is interrupted by any buildings nor has a turn of more than 20 degrees. Continuity is not limited to the selected area and is measured based on GIS maps (Fig. 2). Results are shown by different line-weights, in which the lineweight increases based on its level of continuity. The analysis of continuity is then compared with a complied map of collected cognitive survey results.
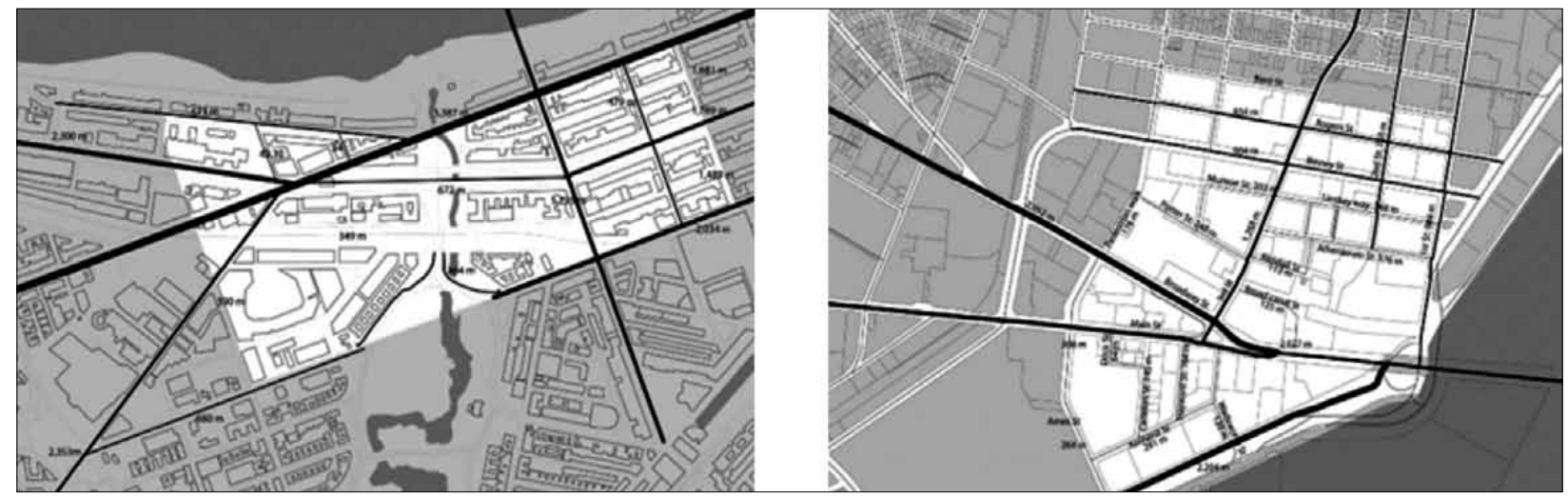

Fig. 2. Objective measurement of continuity. Left: Kenmore and West Back Bay / Right: Kendall Sq. 


\section{Street width}

The second hypothesis suggests that people will remember wider streets more easily than narrow streets. To evaluate this hypothesis on actual street representation, each street width is measured through reading the distance between buildings, where wider streets are shown with a thicker line-weight. To measure the percentage of people's perception, cognitive maps in which each street was drawn is used. As a result, we will compare the width map showing the location of wider streets with compiled cognitive maps to see if a correlation exists.

\section{Average length of street segments}

The third hypothesis suggested that people will remember streets with more number of intersections (density of intersection). To measure this hypothesis, the number of intersections within the length of each street is measured. The average length of street segments for each street is measured by dividing the length of the street by " $n+1$ ", where " $n$ " indicates the number of intersections. The data is then visualized using the thickness of lines, in order to test if streets with smaller average length of segments are remembered more frequently in cognitive maps.

\section{Street patterns}

Measuring street structures is one of the most challenging analyses in this study, since it is difficult to define what street patterns are. Based on street taxonomies that Stephen Marshall provides in categories of linear, tree, radial and cellular patterns (Marshall 2005: 93), we determined two types of street patterns in both Kenmore and Kendall as non-pattern and well-defined pattern. Well-defined patterns can be generated by applying rules to street segments, where we cannot generate specific rules for non-defined street patterns. For instance, grid type and star-shape patterns are examples of well-defined street patterns. The objective map of street patterns is compared with the cognitive map, which is evaluated based on the recognition of well-defined street patterns.

\section{Street angles}

The last hypothesis suggests that people will better remember junctions with less angular deviation from perpendicular angles. Since people are more often used to perpendicular angles, which give them a more clear sense of orientation, they can encode these angles more easily than non-perpendicular angles. The less the deviation from a perpendicular angle is, the better people can remember it. As a result, by using angular deviations measured in the CAD drawing, this hypothesis can be tested as if nodes with smaller angular deviations are the most perceived ones. Whereas the cognitive map explores the percentage of drawings in which perpendicular and non-perpendicular angles are recognized.

\section{Empirical research in Kenmore and Kendall Sq.}

The empirical research is basically the result of compiling the data collected from visual surveys in Kenmore and Kendall Sq. This visual survey asked residents of each area to draw the empty part of the map based on their memory in a scaled plan of Kenmore or Kendall Sq. In order to compile survey results, the researcher counted the number of drawn streets for each street property. Admittedly, this research gained certain benefits from using visual survey methods in form of self-reports as cognitive maps within a snowball sample. The interviewees were found by e-mail through private networks. After receiving replies from the people who reside in the boundaries and were willing to participate in the survey, a fifteen-minute meeting was fixed to complete the map after their oral consent. Figure 3 shows samples of survey results in Kenmore and Kendall Sq.

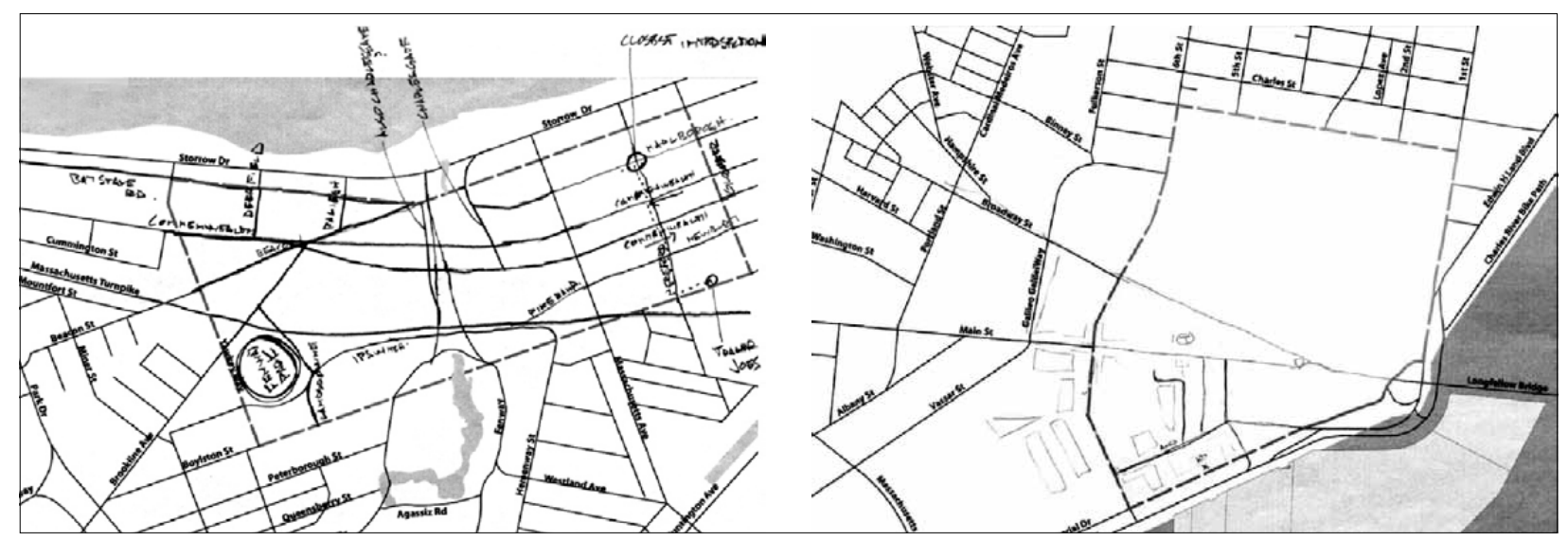

Fig. 3. Sample of survey result in Kenmore and Kendall Sq. 
Survey respondents' statistics indicate that the average age among the selected sample is $25-30$ years with an equal distribution of female and male genders. Approximately 60 percent of the total sample of 30 respondents were raised in urban environments and reside within the boundary for an average of one year in Kenmore and 2.2 years in Kendall Sq. To create cognitive maps based on survey results, compiled maps are generated based on the percentage of "drawn" streets from samples in Kenmore and Kendall Sq. The line weights in compiled maps point to the percentage of perceived streets (Figs 4, 5).

\section{Comparison between actual street analysis and cognitive map results}

\section{Continuity comparison results}

The comparison between the data set for continuity graph results and the percentage of perceived streets shows that there is a correlation between street continuity and people's perception. The scattered diagrams in this part are the result for the compiled data from both Kenmore and Kendall Sq., which gives us a sample size of 30 (Fig. 6).

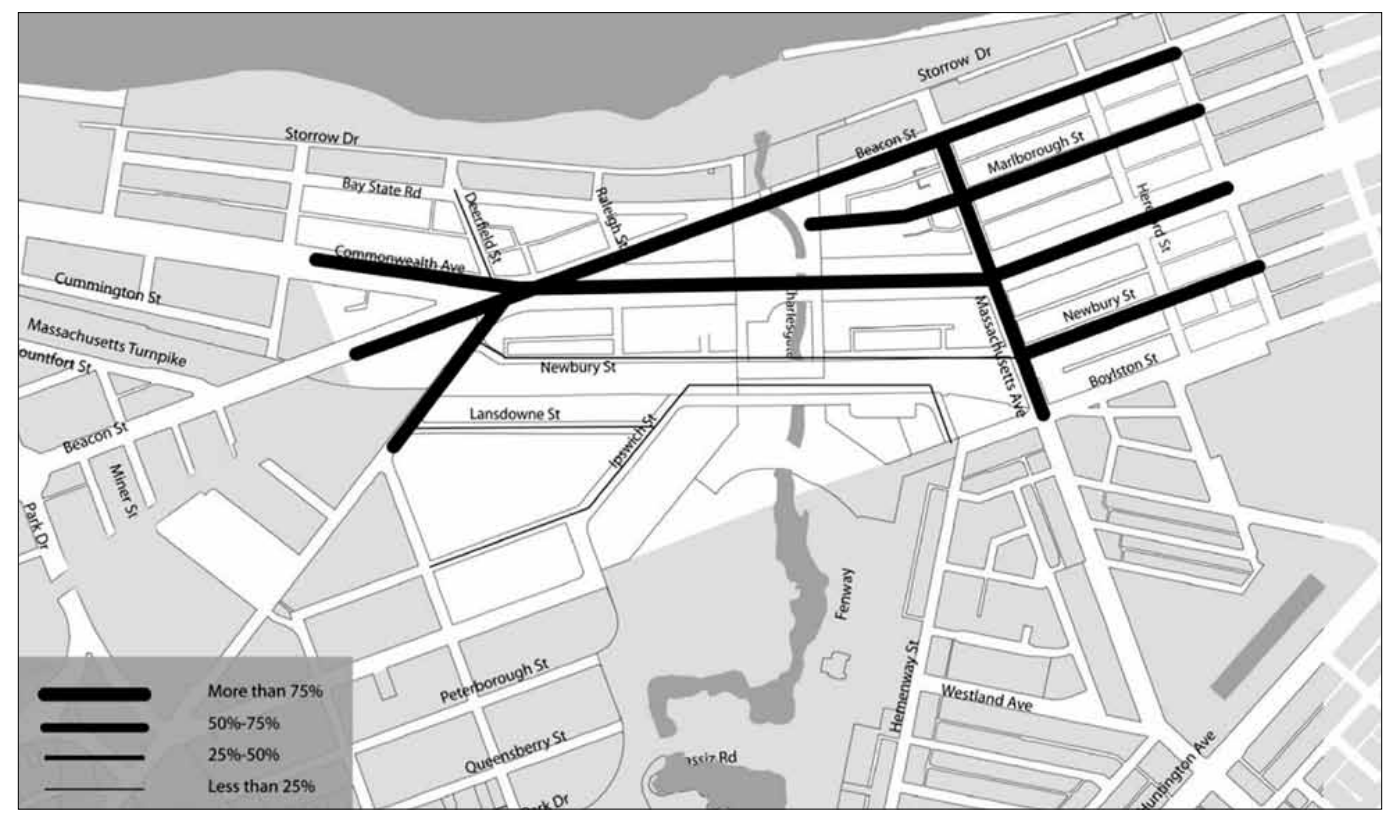

Fig. 4. Kenmore compiled map of perceived streets based on surveys

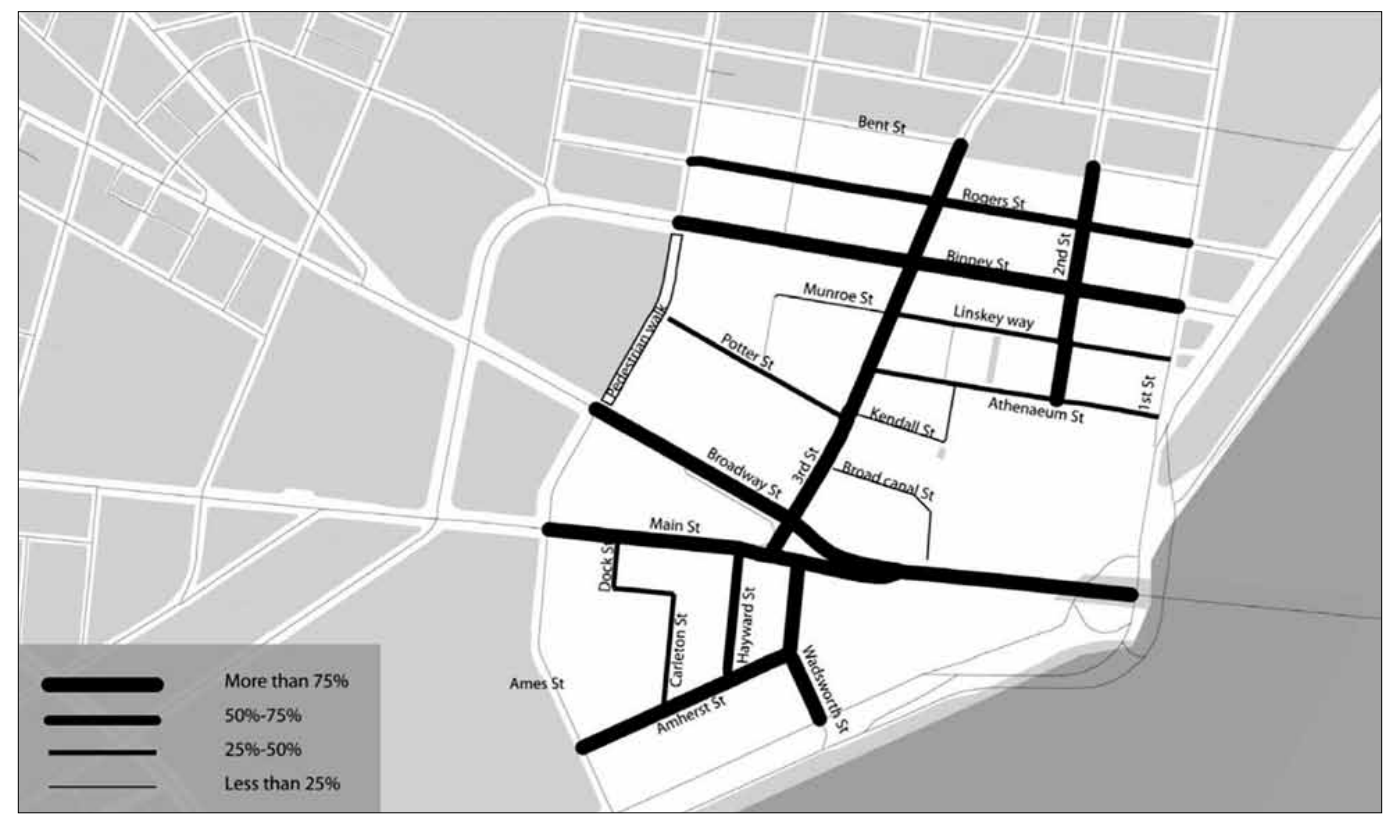

Fig. 5. Kendall Sq. compiled map of accurate perceived streets based on surveys 


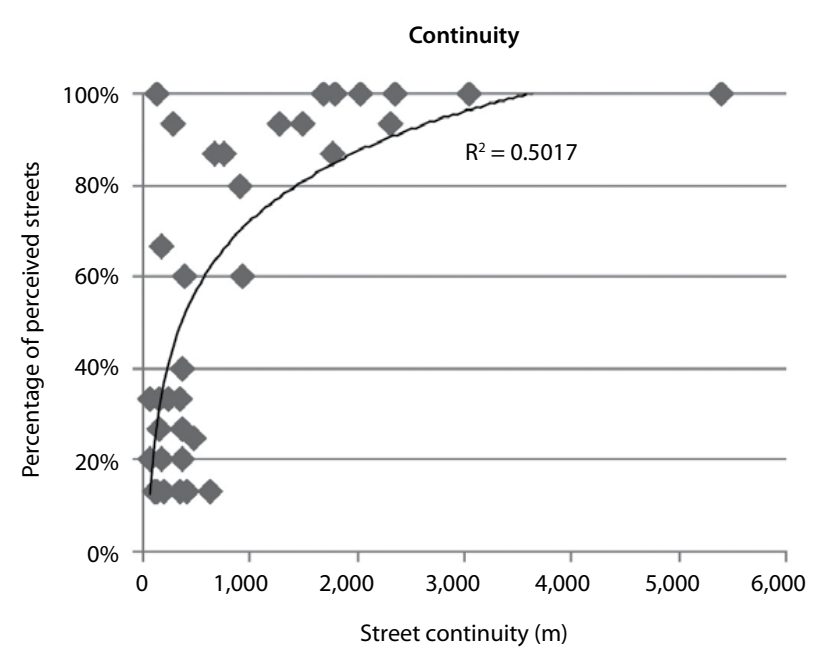

Fig. 6. Comparison chart between street continuity and percentage of perceived streets in Kenmore and Kendall Sq.

\section{Street width comparison results}

The comparison between the street width and percentage of perceived streets shows that these variables are correlated, since the $\mathrm{R}^{\wedge} 2$ of more than 0.3 indicates a considerable relation between the factors. Figure 7 illustrates the correlation between street width and people's perception in Kenmore and Kendall.

Average length of street segments comparison results

This comparison is analyzed based on the correlation between average length of street segments within each boundary and both the percentage of drawn streets and accurate drawn streets. The scattered diagram shows a low and negative relation between them with a $\mathrm{R}^{\wedge} 2$ of 0.02 . As a result, my evidence does not suggest that average length of street segments on a given street affect people's perception (Fig. 8). Moreover, because of a small sample size of 30 , we cannot also reject this hypothesis.

\section{Street pattern comparison results}

Street pattern comparison results show that in both Kenmore and Kendall Sq. people could draw and recognize well-defined patterns with an average of $86.65 \%$ and $93.3 \%$ respectively; however, the number of perceived non-defined streets decreases in both areas. To test if there is a correlation between street patterns and percentage of perceived streets, we assigned 0 to "Nondefined streets" and 1 to "Defined streets." The scattered diagram also indicates a correlation between street patterns and the percentage of perceived streets with the correlation of 0.42 , which shows how well defined street patterns were better perceived. This study, therefore, provided positive results for the fourth hypothesis investigating if people can better remember well-defined street patterns than non-defined structures (Fig. 9).

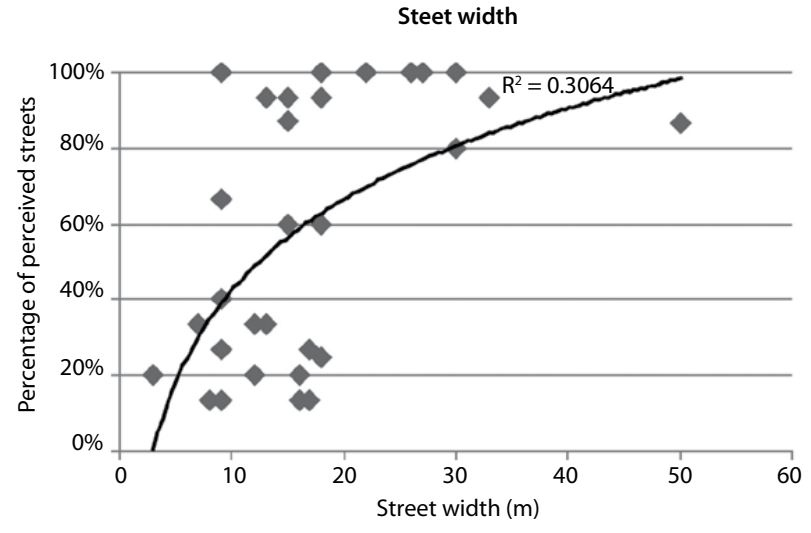

Fig. 7. Comparison chart between street width and percentage of perceived streets in Kenmore and Kendall

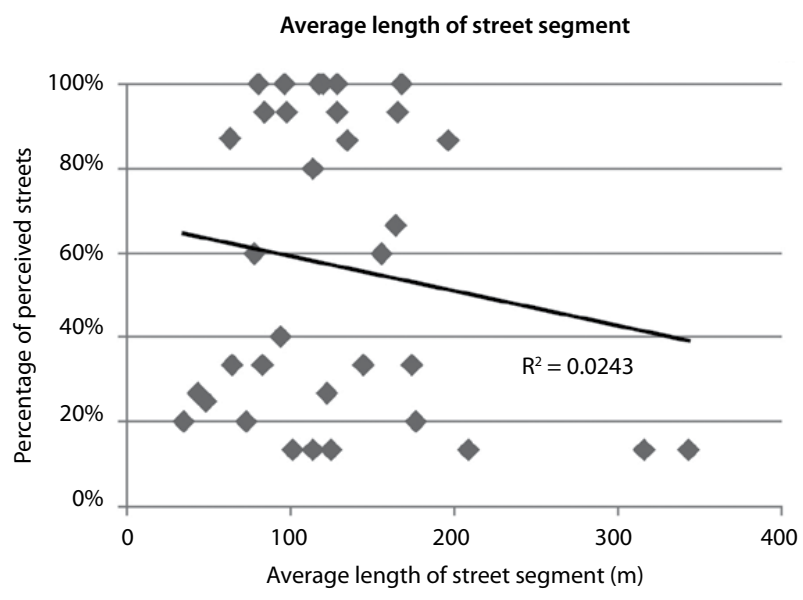

Fig. 8. Comparison chart between average length of street segment and percentage of perceived streets in Kenmore and Kendall Sq.

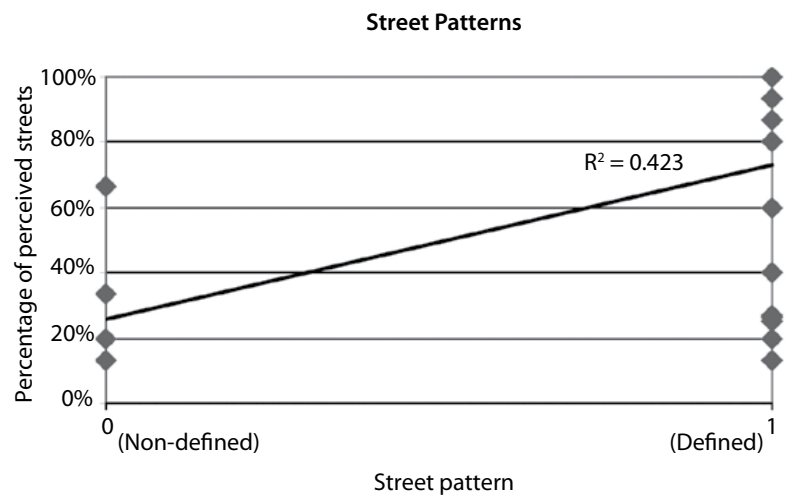

Fig. 9. Comparison chart between street patterns and percentage of perceived streets in Kenmore and Kendall Sq.

\section{Street angles comparison results}

The correlation of street intersections' angular deviation from perpendicular corners appears negative, suggesting that more complex angles are less remembered. This experiment shows that smaller deviations from the perpendicular angles can result in 
better perceptions, while angles which are far from complete angles or junctions with various angular deviations can cause misinterpretations in people's minds. The scattered diagram also represents that deviations more than 45 degrees, which we assumed is difficult to perceive, does not show more than $40 \%$ in people's perception. As a result, the data suggests that this hypothesis about the impact of street angles on people's perception holds (Fig. 10).

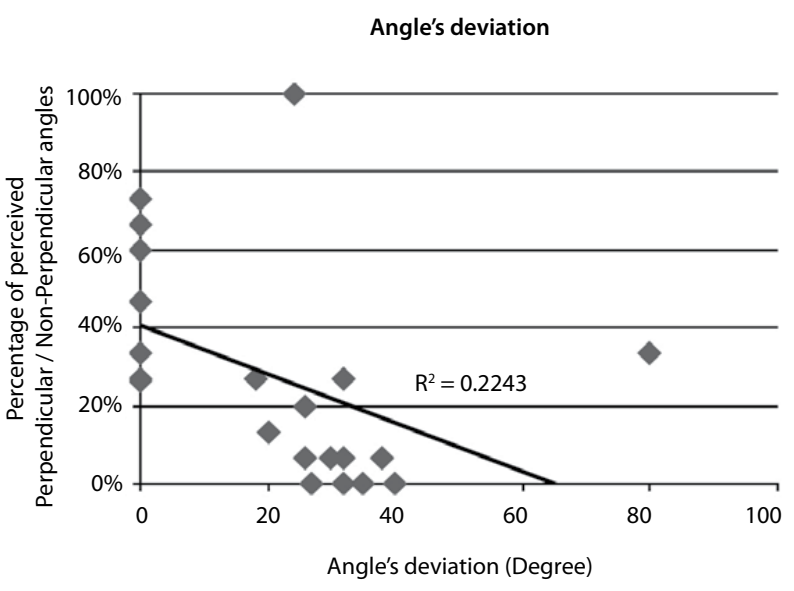

Fig. 10. Comparison chart between street angles and percentage of perceived streets in Kenmore and Kendall Sq.

The correlation plot also addresses another point about the nodes with more number of intersections. As we see, there is a rise in perception axis for two points of Kenmore (80 degrees) and Kendall Sq. (26 degrees), which are the outliers in this analysis. We argue that nodes with a greater number of intersections can be better remembered, as they provide more choices. Since nodes with a greater number of intersections create star-shape patterns, we have also tested this hypothesis in the perception of street patterns. Thus, the results showed that larger deviations from perpendicular angles are less remembered, whereas nodes with a greater number of intersections are better remembered.

\section{Concluding remarks}

This study made an effort to evaluate how different geometric properties of streets impact people's memory and perception of those streets. Kevin Lynch in 1960 argued that paths, edges, districts, nodes and landmarks are the basic elements that formed an environmental image in people's mind, and using these elements evaluated if a city had a better or worse image. Other studies by Jonge in Amsterdam, Appleyard, Stea and Wood (Downs, Stea 1979: 84-5) also followed the same approach to evaluate people's perception based on Lynch's city elements. These pioneering studies initiated a relationship between urban planning and people's perception of a city.

This research claimed that the completeness of the memory of a place demonstrates the extent to what physical patterns of the built environment lend themselves to cognitive representation and therefore explains whether certain types of streets and street patterns are systematically more completely remembered than others. If this is the case, then urban planners might need to develop a much better understanding of how street geometry is perceived by its users. Having extracted street properties influencing people's perception, two sites in Boston were then selected and their actual street properties were compared with the completeness of perceived cognitive maps. To select different geometric characteristics of street networks, street properties such as continuity, width, average length of street segments, street patterns and angles in different intersections are explored. As a result, to evaluate geometric properties of streets, actual street properties were measured and compared with the empirical surveys where residents of corresponding areas were asked to draw their street patterns from memory.

Understanding people's memory and perception of streets requires investigations beyond their geometric analysis, considering how land use and spatial quality of streets could be related to the accuracy of mental maps. Kevin Lynch brought up the idea of urban landmarks, while its impact can be studied under spatial qualities of streets. It is indeed plausible that streets with more pedestrian-oriented activities (shops, restaurants, services, etc.) would be more memorable, and streets that have more people on them would be better remembered. This study is short to compare the impact of geometric properties of streets with the impact of their spatial quality on cognitive maps. However, spatial quality of streets is compared with the cognitive map results to see if any of the existing land use services are better remembered (Fig. 11). Looking at people's drawing results did not support a remarkable influence of street activities on cognitive maps, since quiet streets were also well recalled. As a result, geometry hypotheses can show that despite less popularity, certain geometric attributes of the environment seem to be easier to store in memory, as shown by the evidence in mental map drawings.

The results showed that continuity, street width and pattern had a strong effect on people's perception. We did not find enough evidence to suggest a relationship between average length of street segments and people's perception. Moreover, this study represented that among recognized streets, less deviation from 


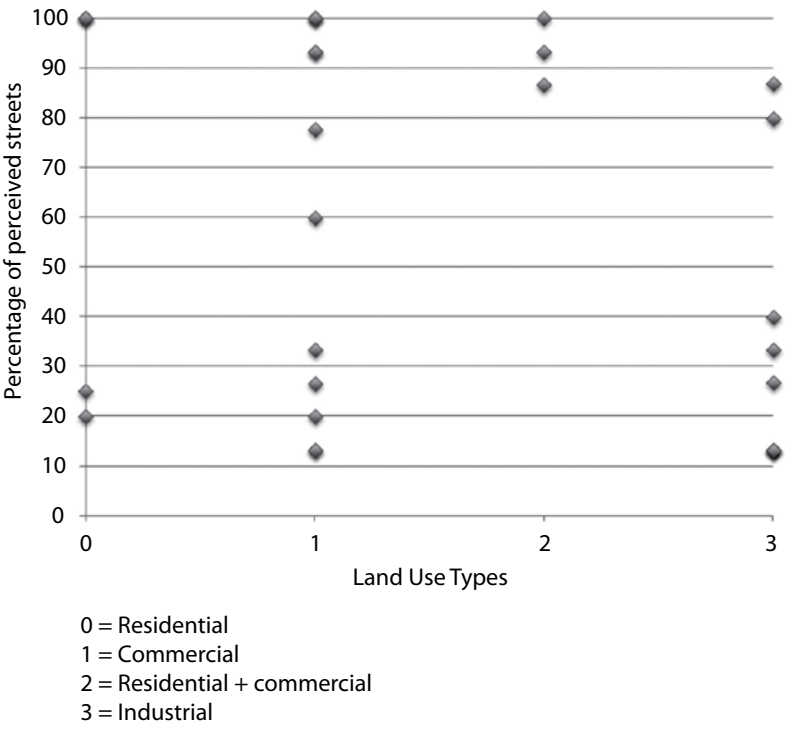

Fig. 11. Comparison chart between street land use services and percentage of perceived streets in Kenmore and Kendall Sq.

perpendicular angles resulted in better understanding and a more clear memory of that intersection. There are, therefore, certain design projections that suggest how to design streets such that they can be more completely remembered. We emphasize, however, that our sample sizes in this study have been too small to suggest a guideline in all these cases and argue that more studies are needed to confirm these effects resulting in analysis guidelines.

\section{Discussions}

Creating places with more complete images in people's minds brings up a profound discussion on the extent to what completeness of memory of a place is beneficial for a city. For instance, New York City is known with its grid-shaped street patterns; however, it is not clear if the completeness of image of New York City is desirable for its residents. Thus, completeness of memory of a place requires a threshold level corresponding to its land use system. In other words, it might be necessary to increase the completeness of memory of a place in residential areas, while a commercial zone would benefit more from street networks that are not easily remembered.

Here are some design implications through the lens of street properties to illustrate the role of street geometries in urban morphology and its configurational pattern. For instance, Cairo is shown in Figure 12a as an example for continuous paths that provide more links to connect shorter paths. This brings me to my next argument about the ways that designers can consider street width. Often, the width of streets is interwoven with their length; however, this street property can solely create a sense of hierarchy and memory. Different street widths are found in the plan of New Delhi represented in Figure 12b. Street patterns, the next street property investigated in this study indicates that welldefined street patterns result in more complete memory of street networks. New Delhi and Washington DC are selected to depict examples of places with well-defined street patterns such as "grids" and "stars" (Figs 12, a, b). This finding could also suggest a design guideline for new urban growth and developments.

Urban development strategies and design disciplines for new urban areas can be affected by guidelines, provided based on configuration studies to create places with more complete images in people's minds. Although, there are different environmental characteristics other than street geometries to improve the level of completeness of cognitive maps, this study tried to control some of them by selecting two areas with similar size, geometrical properties and spatial qualities. a

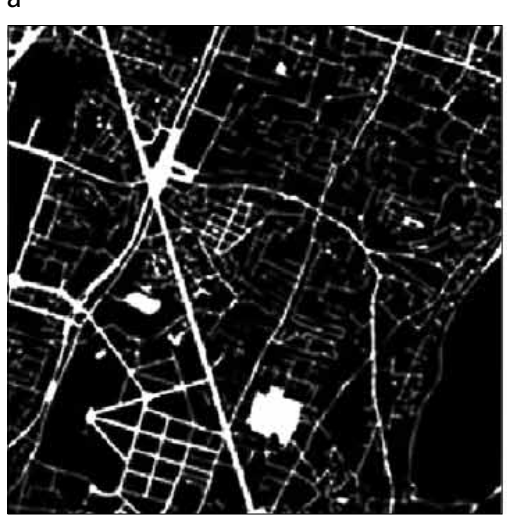

b

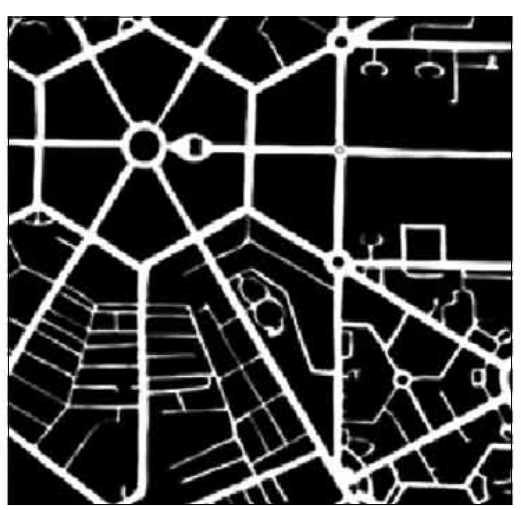

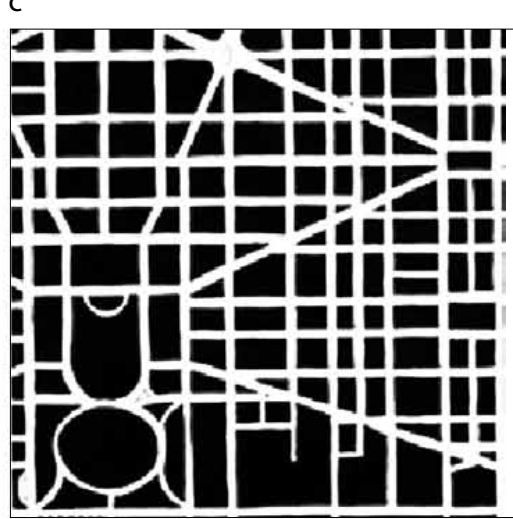

Fig. 12. The geometry of streets in Cairo represents a well-defined example of street continuity (Jacobs 1993: 218) (a); New Delhi signifies an example of well-defined street widths and patterns (Jacobs 1993: 230) (b); Washington DC represents an example of well-defined street patterns (Jacobs 1993: 252) (c) 


\section{Research Limitations}

This research came across several limitations, since it has only dealt with one feature of the built environment as street properties. Hence, this study was short to provide other influential factors such as the impact of land use systems, environmental topography, etc. This research was also limited to five street properties, while other street characteristics can be explored in different areas. For instance, in this research street patterns were limited to two types of grids and starshapes, while these two types do not cover all types of street patterns. The sample size in this study was small and did not distinguish between the respondents who owned a car or pedestrians, who walked everyday. This study, as a result, was limited to suggest design guidelines, where further research in this criterion can result in proposing research guidelines for urban analysts.

\section{References}

Appleyard, D.; Lynch, K.; Myer, J. R. 1964. The view from the road. Cambridge: MIT Press.

Conroy, R. 2003. The secret is to follow your nose: route path selection and angularity, Environment and Behavior 35: 107-131. http://dx.doi.org/10.1177/0013916502238867

Figueiredo, L.; Amorim, L. 2005. Continuity lines in the axial system, in A. van Nes (Ed.). Proceedings of the 5 th International Space Syntax Symposium, 2005 TU Delft, Faculty of Architecture, Delft, 161-174.

Gell, A. 1985. How to read a map: remarks on the practical logic of navigation, Man, New series 20(2): 271-285.

Golledge, R.; Garling, T. 2003. Cognitive maps and urban travel, Research paper No. 601. University of California Transportation Center.

Hilier, B.; Hanson, J. 1984. Social logic of space. Cambridge: Cambridge University Press. http://dx.doi.org/10.1017/CBO9780511597237

Hillier, B. 1996. Space is the machine. Cambridge, New York: Cambridge University Press.

Hölscher, C.; Conroy, D. R.; Turner, A. 2007. Space syntax and spatial cognition, Proceedings of the Workshop held in Bremen 2007 2006, SFB/TR 8 Monographs (2).

Istomin, K.; Dawyer, M. 2009. Finding the way: a critical discussion of anthropological theories of human spatial orientation, Current Anthropology 50(1): 29-49. http://dx.doi.org/10.1086/595624

Jacobs, A. B. 1993. Great streets. Cambridge: MIT Press.

Lynch, K. 1960. The image of the city. Cambridge: MIT Press.

Marshall, S. 2005. Streets \& patterns. London: New York.

Penn, A. 2003. Space syntax and spatial cognition: or why the axial line?, Environment and Behavior 35: 30-65. http://dx.doi.org/10.1177/0013916502238864

Sadalla, E. K.; Montello, D. R. 1989. Remembering changes in direction, Environment and Behavior 21(3): 346-363. http://dx.doi.org/10.1177/0013916589213006

Sanoff, H. 1991. Visual research methods in design. New York: Van Nostrand Reinhold.
Space Syntax [online], [cited 15 November 2010]. Available from Inernet: $w w w . s p a c e s y n t a x . c o m$

Whitehand, J. W. R. 1987. M. R. G. Conzen and the intellectual parentage of urban morphology, Planning History Bulletin 9: 35-41.

\section{MAHSAN MOHSENIN}

MS in Architecture Studies (SMArchS), Massachusetts Institute of Technology.E-mail: mohsenin@alum.mit.edu

Research interests: location theory, urban patterns and landuse distribution, city centers and land-use distribution, daylight patterns at the urban scale.

\section{ANDRES SEVTSUK}

Assistant Professor at Singapore University of Technology and Design, PhD in Urban Design \& Planning, Massachusetts Institute of Technology, Cambridge, MA 02139, USA

E-mail: andres_sevtsuk@sutd.edu.sg

Research interests: urban design, theory of city form, computational spatial, analysis, urban morphology, location theory, urban economics. 